\title{
25 Gbit/s per User Coherent All-Optical OFDM for Tbit/s-Capable PONs
}

\author{
J. Morosi, J. Hoxha, P. Martelli, P. Parolari, G. Cincotti, S. Shimizu, N. Wada, and P. Boffi
}

\begin{abstract}
We propose and test a coherent all-optical orthogonal frequency-division multiplexing (AO-OFDM) system, based on all-optical (de)multiplexing and coherent detection, for multiterabit per second (multi-Tbit/s) passive optical networks (PONs). The proposed approach allows us to greatly increase the downstream data rate per user and extend the standard single-mode fiber reach for future long-reach applications. The compliance with a next-generation PON layout is guaranteed thanks to the exploitation of cyclic arrayed waveguide gratings. Five-user transmission with 12.5 Gbaud quadrature phase shift keying modulation per user $(125 \mathrm{Gbit} / \mathrm{s}$ downstream capacity per wavelength) over a more than $40 \mathrm{~km}$ link has been successfully demonstrated.
\end{abstract}

Index Terms-Fiber-optic communications; OFDM; PON; Waveguide array devices.

\section{INTRODUCTION}

$\mathbf{A}$ $\mathrm{s}$ bandwidth demand keeps on growing due to residential, business, and mobile backhauling traffic, future passive optical networks (PONs) are expected to fully exploit the available spectrum and support very high aggregate throughput. Per-user data rates will increase dramatically, following the exponential request for converged, high-speed, multiple-service applications, and this growth cannot be supported by standard time-division multiplexing (TDM) approaches. Moreover, to increase the network efficiency by node consolidation, PON coverage and transmission distances will also increase considerably [1].

Orthogonal frequency-division multiplexing (OFDM) has been demonstrated as a promising solution for terabit per second (Tbit/s)-capable single wavelength transmission, with high spectral efficiency and high tolerance to chromatic dispersion (CD) effects [2,3]. OFDM superchannels can be generated in the electronic domain, but the maximum achievable throughput is limited by the speed of the digital-to-analog converters that are commercially available [4]. An alternative approach is to generate

Manuscript received November 19, 2015; revised February 4, 2016; accepted February 5, 2016; published March 16, 2016 (Doc. ID 254168).

J. Morosi (e-mail: Jacopo.morosi@polimi.it), P. Martelli, P. Parolari, and P. Boffi are with Politecnico di Milano, Department of Electronics, Information and Bioengineering, via Ponzio 34/5, 20133 Milan, Italy.

J. Hoxha and G. Cincotti are with University Roma Tre, Engineering Department, via Vito Volterra 63, I-00146 Rome, Italy.

S. Shimizu and N. Wada are with National Institute of Information and Communications Technology (NICT), Tokyo, Japan.
OFDM signals in the optical domain, by optically multiplexing independent densely packed OFDM subcarriers. Without using frequency guard bands, all-optical OFDM (AO-OFDM) maximizes the spectral efficiency and overcomes the bandwidth limitations of electronic digital signal processing (DSP) [5,6]. At the transmitter (TX) and receiver (RX) sides, OFDM subcarrier shaping and multiplexing/demultiplexing are achieved by implementing the inverse fast Fourier transform/fast Fourier transform (IFFT/FFT), using only passive and energy-efficient photonic components. Different technologies have been investigated for all-optical IFFT/FFT blocks, based on cascaded Mach-Zehnder interferometers [5], multisection Sagnac interferometers [7], fiber Bragg gratings [8], and arrayed waveguide gratings (AWGs) $[9,10]$. The latter solution presents a simpler planar lightwave circuit layout and reduced insertion losses, and the number of subcarriers is scalable with the number of the device ports.

An alternative solution to demultiplex the OFDM subcarriers is to downconvert a spectral slice of the OFDM superchannel after coherent detection, and to process it in the electrical domain by digital FFT [11]. Coherent detection combined with digital FFT is often adopted in longhaul applications, using DSP circuits operating at very high sampling rates, which are also used to compensate for propagation impairments, to lock phase and frequency, and to demultiplex orthogonal polarizations. However, in AO-OFDM, the OFDM subcarrier shape (that is sinc-like) is wider than the subcarrier spacing, and the bandwidth of the coherent photodetectors and digital sampling strongly influences the subcarrier recovery. The oversampling rate in DSP is also critical to ensure the optimum sampling point [4].

The AO-OFDM architecture, where an AWG at the remote node (RN) is exploited for IFFT operation to demultiplex downstream (DS) subcarriers and to deliver traffic to the different optical network units (ONUs), can instead guarantee the DS throughput required by future-PON medium/large business users. Increased traffic volumes (up to tens of gigabits per second per user) can be handled, overcoming the main limitation of TDM systems. Moreover, the available fiber spectrum can be more efficiently exploited, with respect to conventional wavelength-division multiplexing (WDM) approaches [12]. Hybrid solutions, where the OFDM signal generation is achieved in the electrical domain, are also possible $[13,14]$.

In this kind of AO-OFDM architectures, the AO-OFDM subcarrier spacing, which also equals the modulation rate, 
has to be carefully selected according to network requirements and device limitations. The subcarrier granularity should be fine enough to provide suitable bandwidth to all the users, but the available AWGs, operating as FFT/ IFFT blocks, do not allow us to set the frequency spacing between the neighbor subcarriers lower than $10 \mathrm{GHz}[15-$ 17]. Since narrow frequency spacing is limited by the AWG thermal stability and size, the AO-OFDM subcarrier baud rate remains significantly high and interacts with the channel CD. An AO-OFDM system has already been experimentally demonstrated, using an AWG at both the TX and $\mathrm{RX}$, with a maximum propagation of $15 \mathrm{~km}$ of standard single-mode fiber (SSMF) and 12.5 Gbit/s on-off keying modulation per subcarrier [18].

Coherent detection can be effectively exploited to increase the transmission distance over uncompensated SSMFs in future long-reach PONs. In this case, complex modulation formats combined with polarization-division multiplexing $(\mathrm{PDM})$ can be introduced to increase the capacity per user; moreover, propagation impairments such as CD penalties can be digitally compensated [4].

To the best of our knowledge, coherent AO-OFDM transmission, where the subcarriers are all-optically generated and demultiplexed by suitable photonic devices, has not yet been demonstrated in the literature. In the present paper, we propose an AO-OFDM-based PON, using an AWG at the $\mathrm{RN}$ and coherent detection at the ONU, to achieve a $25 \mathrm{Gbit} / \mathrm{s}$ DS rate per user over a $40 \mathrm{~km}$ uncompensated SSMF link. Moreover, thanks to the cyclic behavior of the AWG at the RN, the proposed AO-OFDM architecture can support a $1.6 \mathrm{Tbit} / \mathrm{s}$ aggregate DS capacity (400 Gbits/s per wavelength), according to the next-generation PON (NG-PON2) requirements, in terms of splitter-based four-wavelength WDM overlay and 64 users [19]. Greater capacity is also achievable using PDM or higher-order modulation formats. Finally, AO-OFDM-based PON architectures can be used advantageously for open access to the infrastructure, assigning a different wavelength to each operator [20].

As a proof of principle, in our experiment, five subcarriers, 12.5 Gbaud quadrature phase shift keying (QPSK)modulated, have been transmitted using a 16-port cyclic AWG. Error-free propagation up to $40 \mathrm{~km}$ SSMF reach has been demonstrated for all the OFDM subcarriers generated and demultiplexed in the all-optical domain, showing 125 Gbit/s DS transmission with negligible penalties, thanks to digital CD compensation. The proposed approach has been shown to be compliant with NG-PON2 requirements in terms of optical distribution network (ODN) losses; moreover, the power budget can be further enhanced by a suitable AWG design in order to target higher capacities and longer distances in view of long-reach PON applications.

\section{AO-OFDM ARCHITECTURE FOR PON NETWORKS}

The proposed AO-OFDM-based PON architecture for DS transmission is shown in Fig. 1. Four different wavelengths that are $200 \mathrm{GHz}$ spaced are used, to match the free

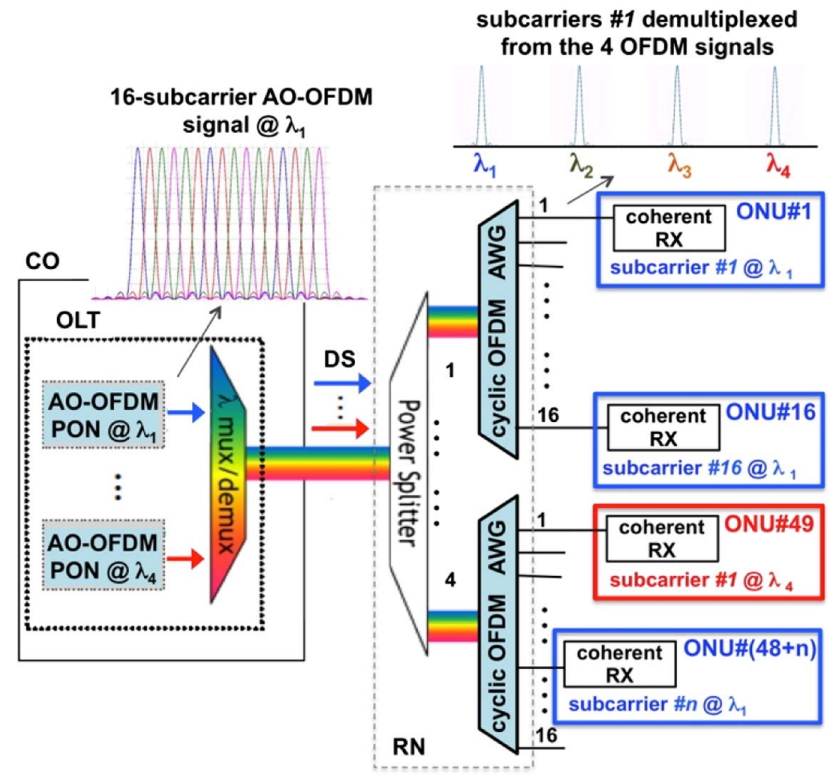

Fig. 1. Coherent AO-OFDM PON system based on the combination of passive splitter and cyclic AWGs at the RN and coherent detection at the ONU. CO, central office; Mux/demux, multiplexer/demultiplexer.

spectral range (FSR) of the cyclic AWG [15,21] employed in the architecture. In this case, a splitter-based overlay is possible, such as in legacy G-PON and XG-PON infrastructures. At the optical line terminal (OLT), a 16-subcarrier $12.5 \mathrm{GHz}$ spaced AO-OFDM signal is generated by implementing the IFFT block in the optical domain for each wavelength. The 1:4 passive splitter at the $\mathrm{RN}$ spreads the aggregate four-wavelength AO-OFDM superchannel toward four parallel cyclic AWGs. Each AWG implements the FFT function, and at the same time demultiplexes the AO-OFDM subcarriers and the four wavelengths. The AWG is connected to $16 \mathrm{ONUs}$ via drop fibers, so that the DS signal is shared among 64 ONUs, according to NG-PON2 requirements. Compared to a passive 1:64 splitter characterized by minimum $18 \mathrm{~dB}$ insertion losses, the cascade of a 1:4 splitter and the AWG allows us to increase the transmission power budget and thus to target longer reaches.

The use of coherent detection at each ONU combined with the passive demultiplexing of the OFDM subcarriers performed by the AWG offers the opportunity to increase the user data rate on request, meeting the NG-PON2 requirements and also allowing multiple-service aggregation. Multilevel modulation formats can be used to flexibly increase the DS user data rate without changing the system architecture; furthermore, PDM can be also introduced to further double the system capacity. Using 12.5 Gbaud QPSK modulation for each subcarrier, a 64user PON achieves a $1.6 \mathrm{Tbit} / \mathrm{s}$ aggregate DS capacity and $2 \mathrm{bit} / \mathrm{Hz}$ spectral efficiency for each wavelength. The transported rate becomes 3.2 Tbits/s if PDM is used.

Furthermore, coherent detection provides information on the amplitude and phase of the received signals and 
allows postprocessing algorithms to compensate linear propagation impairments, such as $\mathrm{CD}$, and to equalize the signal with respect to the channel. These operations are required in particular in long-reach PONs with high ODN losses, and when high-order modulation formats are used.

On the other hand, the cyclic AWG is exploited to send four subcarriers at different wavelengths to each ONU; using a tunable local oscillator, each ONU selects the target wavelength. This approach allows open access to the infrastructure among different operators.

Finally, all-optical demultiplexing performed by the AWG before detection relaxes the requirements of the coherent RXs in terms of DSP complexity, because the FFT is performed in the optical domain. Moreover, the oversampling rate and $\mathrm{RX}$ bandwidth can be reduced with respect to conventional approaches. Multi-Tbit/s aggregate DS capacity can be achieved, also guaranteeing flexibility in resource allocation.

The DS transmission is analyzed in detail in the following sections. On the other hand, the upstream (US) transmission can rely on a solution based on ultradense WDM [22] by exploiting the same ODN described in Fig. 1. The RN AWG is employed to multiplex the US WDM carriers spaced $12.5 \mathrm{GHz}$ apart, generated by the ONUs. The bit rate of the US data is chosen to guarantee no spectral overlapping between the WDM carriers after the WDM multiplexing [13]. Thus, the PON architecture is not symmetric, and the US capacity is reduced with respect to the OFDM-based DS.

\section{EXPERIMENTAL SETUP}

We demonstrate the feasibility of the coherent AOOFDM-based PON described in Section II, considering five all-optically generated subcarriers, QPSK-modulated at 12.5 Gbaud, in order to achieve a $25 \mathrm{Gbit} / \mathrm{s}$ equivalent DS data rate per user $(125 \mathrm{Gbits} / \mathrm{s}$ DS raw data per wavelength). Figure 2 shows the experimental setup used to generate the five orthogonal phase-locked $12.5 \mathrm{GHz}$ spaced AO-ODFM subcarriers, using a wavelength-selective switch

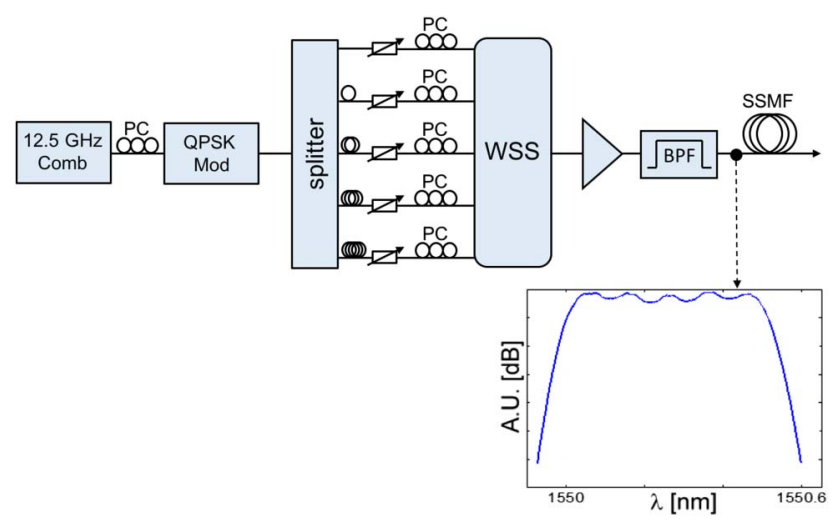

Fig. 2. Transmitter setup for AO-OFDM signal generation. BPF, band-pass filter. Inset: measured optical spectrum at the WSS output.
(WSS) and a flat and stable optical comb. The optical comb is generated by the cascade of a tunable laser, a $12.5 \mathrm{GHz}$ clock-driven Mach-Zehnder modulator, a $12.5 \mathrm{GHz}$ clockdriven optical phase modulator, a SSMF compressing span, and finally an optical delay line synchronizing the optical pulse peaks with the electrical signal, as explained in detail in [21]. In the experiment we have replaced the AWG at the TX with a WSS, with a second-order super-Gaussian transfer function, to provide suitable signal shaping and flexible subcarrier multiplexing [23]. The optical comb lines are QPSK modulated by two $2^{15}-1$ pseudorandom bit sequences at 12.5 Gbaud, and the five central flat subcarriers are extracted by means of suitable splitting. Optical delay lines of different lengths are used to guarantee full data decorrelation at the WSS inputs. Variable optical attenuators (VOAs) and polarization controllers (PCs) allow us to equalize subcarrier powers while minimizing the cross talk.

The superchannel spectrum constituted by five all-optically generated OFDM subcarriers measured at the WSS output is shown in the inset of Fig. 2. The total transmitted power is $2 \mathrm{dBm}$. The OFDM superchannel propagates over $40 \mathrm{~km}$ uncompensated SSMF, which corresponds to the feeder fiber of the PON. The RX schematic is reported in Fig. 3(a).

The OFDM signal is demultiplexed by a 16-port AWG performing the FFT operation in a passive and all-optical way. The measured transfer function of the $12.5 \mathrm{GHz}$ spaced AWG is shown in Fig. 3(b). As can be seen, at each AWG output port (corresponding to a particular color in the figure), the cyclic channels are spaced $200 \mathrm{GHz}$ apart (corresponding to the AWG FSR), and in our present implementation the AWG shows nearly $10 \mathrm{~dB}$ losses.

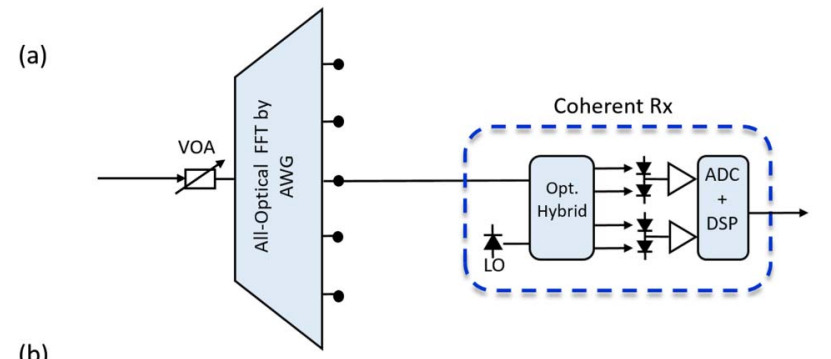

(b)

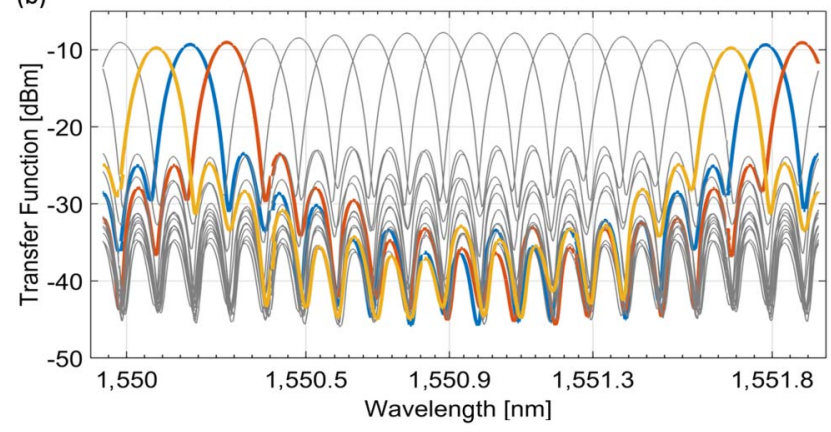

Fig. 3. (a) Receiver setup for AO-OFDM passive demultiplexing and the coherent detection, (b) measured cyclic sinc-like AWG transfer function, with $1612.5 \mathrm{GHz}$ spaced subchannels. The curve at the same color shows the cyclic AWG behavior at each port with $200 \mathrm{GHz}$ FSR. ADC, analog-to-digital converter. 
Before the AWG, a VOA introduces fixed $6 \mathrm{~dB}$ losses, and emulates the passive 1:4 splitter of the PON architecture presented in Fig. 1. The tested whole $16 \mathrm{~dB}$ losses allow us to achieve a $2 \mathrm{~dB}$ increase in the budget with respect to the 1:64 splitter exploitation, even when employing such a high-loss AWG. After passive demultiplexing, each single subcarrier is coherently received, using a tunable laser source as the local oscillator. No PDM is used in this preliminary experimentation. The signal is acquired by means of a Tektronix real-time oscilloscope with a 50 GSample/s sampling rate, then processed offline.

\section{EXPERIMENTAL RESULTS}

Figure 4 presents the optical spectra measured at different AWG output ports, showing the all-optical subcarrier demultiplexing. The demultiplexed central subcarrier spectrum is shown in Fig. 4(a.1), when all the other OFDM subcarriers have been also transmitted in back-to-back (BTB). The spectrum at the same AWG port, when only the central subcarrier is switched off, is shown in Fig. 4 (a.2); we observe that the cross talk due to the neighbor subcarriers is almost negligible ( $29 \mathrm{~dB}$ lower). The spectrum of the demultiplexed $25 \mathrm{GHz}$ spaced subcarrier with respect to the central one is shown in Fig. 4(b.1), whereas Fig. 4(b.2) shows the signal at the same AWG output port when that corresponding subcarrier is switched off. In the latter case, we observe significant cross talk $(-13 \mathrm{~dB})$ due to the limited extinction of the AWG.

Figure 5 reports the bit error rate (BER) measurements versus the received optical power per subcarrier for all five
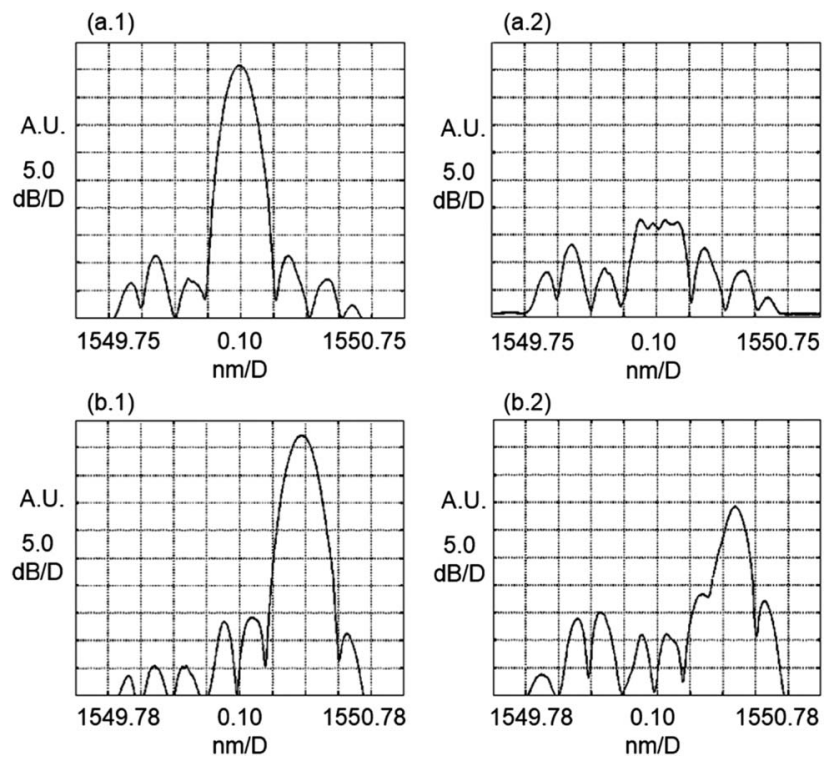

Fig. 4. (a.1) Spectrum of the demultiplexed central subcarrier when all the other OFDM $12.5 \mathrm{GHz}$ spaced subcarriers are transmitted in BTB, (a.2) spectrum at the same AWG output port when the central subcarrier is switched off (cross talk), (b.1) spectrum of the demultiplexed $25 \mathrm{GHz}$ spaced subcarrier, (b.2) spectrum at the same output port when the $25 \mathrm{GHz}$ spaced subcarrier is switched off (cross talk).

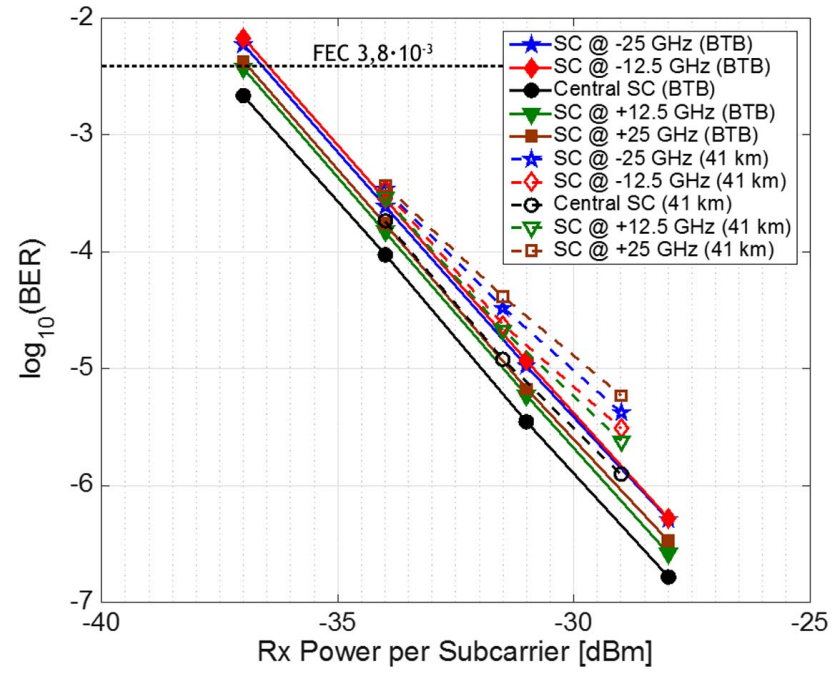

Fig. 5. Measured BER versus received power per subcarrier for the five 25 Gbit/s QPSK AO-OFDM subcarriers in BTB (solid lines) and after $41 \mathrm{~km}$ SSMF propagation and digital CD compensation (dashed lines).

demultiplexed $12.5 \mathrm{GHz}$ spaced AO-OFDM subcarriers, both in BTB (solid lines) and after uncompensated propagation along $41 \mathrm{~km}$ SSMF (dashed lines). A tunable local oscillator is used at the coherent RX. In BTB we observe that each $25 \mathrm{Gbit} / \mathrm{s}$ QPSK subcarrier coherently detected presents a similar performance, with a worse performance for subcarriers corresponding to limited-extinction AWG ports. In the case of $41 \mathrm{~km}$ SSMF propagation, coherent detection allows us not only to receive multilevel modulation formats, such as QPSK, but also to digitally compensate for CD. Therefore, the system performance shows very small penalties with respect to BTB. With an optical received power per subcarrier of $-34 \mathrm{dBm}$, a BER lower than a $7 \%$ overhead forward error correction (FEC) threshold of $3.8 \cdot 10^{-3}$ [24] is achieved for all the subcarriers, sustaining ODN losses higher than $29 \mathrm{~dB}$, according to the NG-PON2 requirements. In the tested $41 \mathrm{~km}$ SSMF propagation, taking into account the FEC threshold, the ODN losses allow a $5 \mathrm{~dB}$ power margin, which can be exploited to increase the PON reach. Moreover, the tested AWG is a first prototype, whose insertion losses are expected to be reduced by around $5 \mathrm{~dB}$ in its final implementation. Thus, the proposed architecture is capable of bridging up to $80 \mathrm{~km}$ SSMF, as CD is compensated thanks to the coherent detection.

\section{Conclusions}

We have proposed for the first time (to our knowledge) a coherent AO-OFDM-based PON, using coherent detection at the ONU and an AWG at the RN to cyclically demultiplex wavelengths and FDM subcarriers in a passive way. The main advantages of the proposed architecture are: 
- a flexible increase in the DS rate per user, using highlevel modulation formats and also PDM, which can be introduced on request;

- use of a tunable local oscillator at the ONU to select a different wavelength, for an unbundled operation in case of multiple operators;

- increased ODN reach, reduction of the ODN losses with respect to the 1:64 splitter-based overlay, and digital compensation for linear propagation impairments, such as CD;

- no digital FFT implementation to demultiplex the OFDM subcarriers.

The overall DSP computation complexity at the ONU is thus driven by the CD compensation, requiring a number of digital taps proportional to the transmission distance to be compensated [25] (tens of kilometers for the considered PONs). The related number of operations is significantly less than that required to perform the FFT, given 16 OFDM subcarriers.

In particular, we have experimentally demonstrated the feasibility of the proposed architecture in the case of fiveuser 12.5 Gbaud AO-OFDM transmission with QPSK modulation. The OFDM superchannel, all-optically generated and multiplexed, has a $125 \mathrm{Gbit} / \mathrm{s}$ capacity per wavelength. A 16-port cyclic AWG has been used at the RN for FFT implementation and subcarrier demultiplexing in the optical domain. Propagation over more than $40 \mathrm{~km}$ SSMF has been demonstrated without significant penalties for all the subcarriers. The proposed architecture allows multiTbit/s aggregate DS capacity, using four wavelengths and connecting 64 users, according to NG-PON2 standards.

Considering the BER performance demonstrated at $-34 \mathrm{dBm}$ optical received power per subcarrier, in our experiment the power budget would allow us to increase the propagation beyond $40 \mathrm{~km}$, CD being digitally compensated after the coherent detection.

Thanks to a new suitable OFDM AWG design with limited losses compliant with those provided by standard WDM AWGs, and to the tested power budget margins, higher-order modulation formats could be effectively exploited to achieve very-high-capacity PONs or long-reach transmission.

\section{ACKNOWLEDGMENT}

The authors thank TEKTRONIX for the experiment support. This work was supported in part by the Italian Ministry of University and Research through the ROADNGN project (PRIN2010-2011) and by the EU Seventh Framework Programme (FP7/2007-2013) through the ASTRON project (grant agreement no. 318714).

\section{REFERENCES}

[1] S. McGettrick, F. Slyne, N. Kitsuwan, D. B. Payne, and M. Ruffini, "Experimental end-to-end demonstration of shared $\mathrm{N}: 1$ dual homed protection in long reach PON and SDN-controlled core," in Proc. OFC, 2015, paper Tu2E.5.

[2] Y. Ma, Q. Yang, Y. Yang, S. Chen, and W. Shieh, "1-Tb/s singlechannel coherent optical OFDM transmission over $600-\mathrm{km}$
SSMF fiber with subwavelength bandwidth access," Opt. Express, vol. 17, pp. 9421-9427, 2009.

[3] X. Yi, N. K. Fontaine, R. P. Scott, and S. J. B. Yoo, “Tb/s coherent optical OFDM systems enabled by optical frequency combs," J. Lightwave Technol., vol. 28, pp. 2054-2061, 2010.

[4] Y. K. Huang, E. Ip, Z. Wang, M. F. Huang, Y. Shao, and T. Wang, "Transmission of spectral efficient super-channels using all-optical OFDM and digital coherent receiver technologies," J. Lightwave Technol., vol. 29, pp. 3838-3844, 2011.

[5] A. J. Lowery and L. B. Du, "All-optical OFDM transmitter design using AWGRs and low-bandwidth modulators," Opt. Express, vol. 19, pp. 15696-15704, 2011.

[6] R. Schmogrow, T. Schellinger, M. Jordan, M. Winter, G. Huber, T. Vallaitis, R. Bonk, P. Kleinow, F. Frey, M. Roeger, S. Koenig, A. Ludwig, A. Marculescu, J. Li, M. Hoh, M. Dreschmann, J. Meyer, S. Ben Ezra, N. Narkiss, B. Nebendahl, F. Parmigiani, P. Petropoulos, B. Resan, A. Oehler, K. Weingarten, T. Ellermeyer, J. Lutz, M. Moeller, M. Huebner, J. Becker, C. Koos, W. Freude, and J. Leuthold, " 26 Tbit/s ${ }^{-1}$ line-rate superchannel transmission utilizing all-optical fast Fourier transform processing," Nat. Photonics, vol. 5, pp. 364-371, 2011.

[7] J. Du and C. Shu, "Cascaded and multisection Sagnac interferometers for scalable and tunable all-optical OFDM DEMUX,” J. Lightwave Technol., vol. 31, pp. 2307-2313, 2013.

[8] H. Chen, M. Chen, and S. Xie, "All-optical sampling orthogonal frequency-division multiplexing scheme for high-speed transmission system," J. Lightwave Technol., vol. 27, pp. 4848-4854, 2009.

[9] A. J. Lowery, "Design of arrayed-waveguide grating routers for use as optical OFDM demultiplexers," Opt. Express, vol. 18, pp. 14129-14143, 2010.

[10] S. Shimizu, G. Cincotti, and N. Wada, "Demonstration and performance investigation of all-optical OFDM systems based on arrayed waveguide grating," Opt. Express, vol. 20, pp. B525-B534, 2012.

[11] S. Chandrasekhar and X. Liu, "Experimental investigation on the performance of closely spaced multi-carrier PDM-QPSK with digital coherent detection," Opt. Express, vol. 17, pp. 21350-21361, 2009.

[12] L. Marazzi, M. Brunero, M. Martinelli, R. Brenot, A. Maho, S. Barbet, G. Gavioli, G. Simon, S. Le, F. Saliou, and P. Chanclou, "C- and O-band operation of RSOA WDM PON self-seeded transmitters up to $10 \mathrm{~Gb} / \mathrm{s}$ [Invited]," J. Opt. Commun. Netw., vol. 7, A249-A255, 2015.

[13] P. Boffi, P. Martelli, P. Parolari, L. Blundetto, J. Morosi, and G. Cincotti, "Demonstration and performance investigation of hybrid OFDM systems for optical access network applications," IEEE Photonics J., vol. 7, 6987242, 2015.

[14] J. Morosi, P. Boffi, P. Martelli, P. Parolari, G. Cincotti, S. Shimizu, and N. Wada, "Hybrid OFDM experimentation based on arrayed waveguide grating for all-optical FFT implementation in PON architecture," Electron. Lett., vol. 51, pp. 1802-1804, 2015.

[15] X. Wang, N. Wada, N. Kataoka, T. Miyazaki, G. Cincotti, and K.-J. Kitayama, " $100 \mathrm{~km}$ field trial of $1.24 \mathrm{Tbit} / \mathrm{s}$, spectral efficient, asynchronous $5 \mathrm{WDM} \times 25$ DPSK-OCDMA using one set of 50'50 ports large scale en/decoder," in Proc. OFC, 2007, paper PDP14.

[16] N. K. Fontaine, R. P. Scott, J. H. Baek, X. Zhou, T. Su, S. Cheung, Y. Wang, C. Junesand, S. Lourdudoss, K. Y. Liou, R. A. Hamm, W. Wang, B. Patel, L. A. Gruezke, W. T. Tsang, J. P. Heritage, and S. J. B. Yoo, "Monolithic InP 100 -channel $\times 10-\mathrm{GHz}$ device for optical arbitrary waveform generation," IEEE Photonics J., vol. 3, pp. 975-985, 2011. 
[17] S. Shimizu, G. Cincotti, and N. Wada, "Demonstration of $8 \times 12.5 \mathrm{Gbit} / \mathrm{s}$ all-optical OFDM system with an arrayed waveguide grating and waveform reshaping," in Proc. ECOC, 2012, paper Th.1.A.2.

[18] S. Shimizu, G. Cincotti, and N. Wada, "Chromatic dispersion monitoring and adaptive compensation using pilot symbols in an $8 \times 12.5 \mathrm{Gbit} / \mathrm{s}$ all-optical OFDM systems," Opt. Express, vol. 22, pp. 8734-8741, 2014.

[19] "40-Gigabit-capable passive optical networks 2 (NG-PON2): Physical media dependent (PMD) layer specification," ITUT Recommendation G.989.2, Dec. 2014.

[20] A. Dixit, M. Van der Wee, B. Lannoo, D. Colle, S. Verbrugge, M. Pickavet, and P. Demeester, "Fiber and wavelength open access in WDM and TWDM passive optical networks," IEEE Network, vol. 28, pp. 74-82, 2014.
[21] J. Hoxha, J. Morosi, S. Shimizu, P. Martelli, P. Boffi, N. Wada and G. Cincotti, "Spectrally-efficient all-optical OFDM by WSS and AWG," Opt. Express, vol. 23, pp. 10986-10996, 2015.

[22] M. Presi, R. Corsini, M. Artiglia, and E. Ciaramella, "Ultradense WDM-PON 6.25 GHz spaced $8 \times 1 \mathrm{~Gb} / \mathrm{s}$ based on a simplified coherent-detection scheme," Opt. Express, vol. 23, pp. 22706-22713, 2015.

[23] Z. Xu, X. Cheng, Y.-K. Yeo, X. Shao, L. Zhou, and H. Zhang, "Large-scale WDM passive optical network based on cyclical AWG," Opt. Express, vol. 20, pp. 13939-13947, 2012.

[24] T. Mizuochi, "Next generation FEC for optical communication," in Proc. OFC, 2008, paper OTuE5.

[25] G. Goldfarb and G. Li, "Chromatic dispersion compensation using digital IIR filtering with coherent detection," IEEE Photonics Technol. Lett., vol. 19, pp. 969-971, 2007. 